\title{
A Complementary LC-tank Based IR-UWB Pulse Generator for BPSK Modulation
}

\author{
Iman Kianpour, Bilal Hussain and Vítor G. Tavares \\ INESC-TEC and Faculty of Engineering, University of Porto, Portugal \\ \{mapt1209340, bhussain,vgt\}@fe.up.pt
}

\begin{abstract}
This paper presents a low-power binary phase shift keying (BPSK) pulse generator for ultra-wide-band transmitters. The circuit has been designed based on LC-tank resonators using 0.13 um CMOS technology. Simulation shows $-10 \mathrm{~dB}$ bandwidth of around $3 \mathrm{GHz}$ and power consumption of $2 \mathrm{~mW}$ at $100 \mathrm{MHz}$ PRF. Peak-peak amplitude voltage for both symbols ' 1 ' and ' $O$ ' are approximately as large as $1.2 \mathrm{~V}$ supply voltage and can radiate enough energy to satisfy the FCC mask only by one pulse. Thus, the energy consumption is $20 \mathrm{pJ} /$ pulse/bit. Pulse duration is $1.5 \mathrm{~ns}$ and the transmitter can reach data rates of $660 \mathrm{Mbps}$
\end{abstract}

\section{Introduction}

UWB frequency range (3.1-10.6 GHz) was released and defined by the Federal Communications Commission (FCC) in 2002 as an unlicensed frequency band at a limited Effective Isotropic Radiated Power (EIRP) of $-41.3 \mathrm{dBm} / \mathrm{MHz}$ in order not to interfere with narrow-band systems like GPS, Personal Cell Systems (PCS), Bluetooth, etc. [1], [2], [3]. After a decade, this frequency range has lost its interests for mobile communication mostly due to limited transmission power/range. However, still it can be used in other specific applications, e.g. wireless sensor networks (WSN), biomedical implants and internet-ofthings (IoT).

Impulse-Radio UWB (IR-UWB) is one of the most suitable communication techniques for UWB lowpower applications, which uses a short-duration pulse with large bandwidth and known as a carrier-less technique. Not using up and down conversion, IRUWB transmitters have low complexity in architecture, leading to less power consumption, less die area and lower production cost. These properties make UWB transceivers eminently suitable for sensor nodes in IoT and WSN [2], [4].

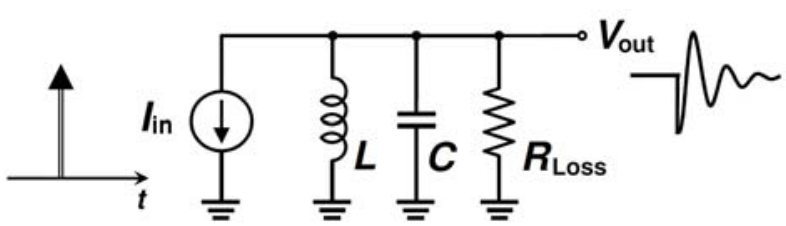

Figure 1. Decaying oscillatory behavior of a lossy LC tank triggered by a current impulse [6]

Different modulation techniques can be used in IRUWB transmitters, such as binary phase-shift keying (BPSK), pulse-position modulation (PPM), and on-off keying (OOK). BPSK (a.k.a. biphasic) is suitable for smooth spectrum usage and it has a better bit error rate (BER), but usually channel estimation is required and the architecture of the system becomes large and complex due to coherence requirement. Contrarily, OOK and PPM are based on the presence and absence of the pulse and no channel estimation is required. Compared to BPSK, OOK and PPM have less BER [4], [5].

A variety of techniques have been used to design IR-UWB BPSK pulse generators, from all-digital techniques [7] to LC-VCO based [8], [9] and mixer based circuits [10], [11]. The work introduced in [12] has used two ring oscillators triggered by positive and negative signals. Combination of delayed triangular glitches [13] and Step Recovery Diode (SRD) based circuits [14] also have been presented in literature. Another approach is to apply a glitch (spike) to a filter network [15], [16], [17] such that it forms a UWB impulse at the output; however, using complex filters and drivers has led to higher power consumption. In [16], although $1.93 \mathrm{pJ}$ emitted energy per pulse is reported, the circuit dissipates $393 \mathrm{pJ}$ to generate a single pulse. The very last technique also can be seen as usage of decaying oscillatory behavior of an RLC network, which is the fundamental idea used by the proposed IR-UWB pulse generator in this paper.

The rest of this paper is organized as follows. In section II, the proposed pulse generator has been 
introduced. In section III, simulation results will be discussed, followed by a conclusion in section IV.

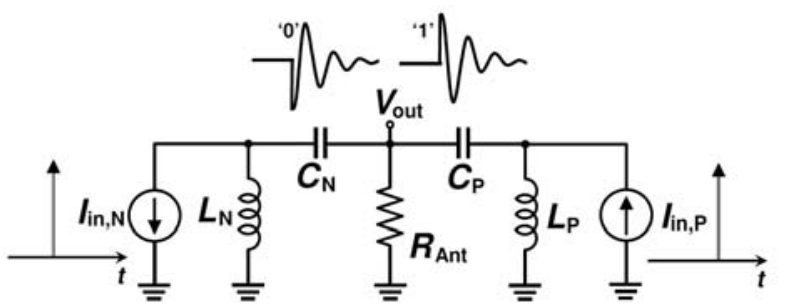

Figure 2. A complementary circuit can invert the direction of impulse so that the polarity of the output voltage will be reversed.

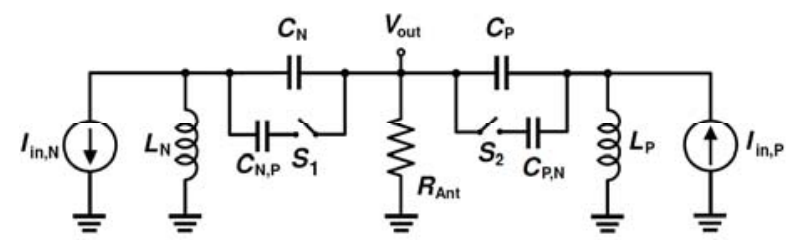

Figure 3. A complementary tank can be used as a filter for $1.6 \mathrm{GHz}$ frequency if the value of the capacitor changes.

\section{Proposed Pulse Generator}

As illustrated in Fig. 1, a lossy LC tank triggered by a current impulse shows a decaying oscillatory response. In fact, the resistance of the capacitor and the inductor converts into heat some of the energy that reciprocates between them. This concept can be used for designing a very low power pulse generator, mainly because there are not many components in such a circuit. The UWB pulse generator is supposed to drive a $50 \mathrm{ohm}$ antenna, therefore in the RLC network the $\mathrm{R}_{\text {Loss }}$ can be replaced by $\mathrm{R}_{\text {Ant }}=50 \mathrm{ohm}$. In power spectral density (PSD), the central frequency is defined as $\omega_{0}=1 / \operatorname{sqrt}(\mathrm{LC})$ and how fast or slow the signal decays will determine the bandwidth. The faster it decays, the wider bandwidth will be obtained.

By adding a complementary circuit (Fig. 2), the direction of the current through $\mathrm{R}_{\text {Ant }}$ can be reversed so that it creates a pulse with opposite phase that serves BPSK modulation. The capacitor comes in series in the RLC network in order to better filter out the low frequency components of the input impulse. In the positive half circuit including $I_{i n, P}, L_{P}$ and $C_{P}$, the complementary negative LC tank, including $\mathrm{L}_{\mathrm{N}}$ and $\mathrm{C}_{\mathrm{N}}$, would easily kill the signal because it oscillates at the same frequency. Yet, they can be seen as a series LC filter to suppress the frequencies around $1.6 \mathrm{GHz}$ where FCC rules are more strict because of satellite and GPS band [1]. Thus, the capacitance $\mathrm{C}_{\mathrm{N}}$ should increase by using a larger capacitor $\mathrm{C}_{\mathrm{N}, \mathrm{P}}$ coming in

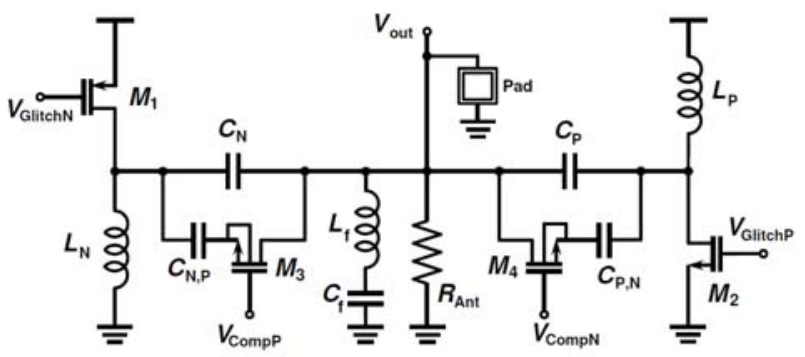

(a)

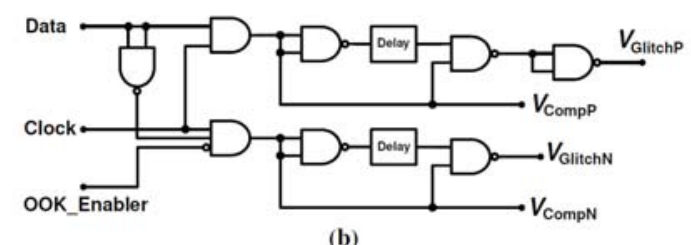

(b)

Figure 4. Proposed IR-UWB modulator. (a) Complementary pulse generator, (b) Control signals and glitch generator.

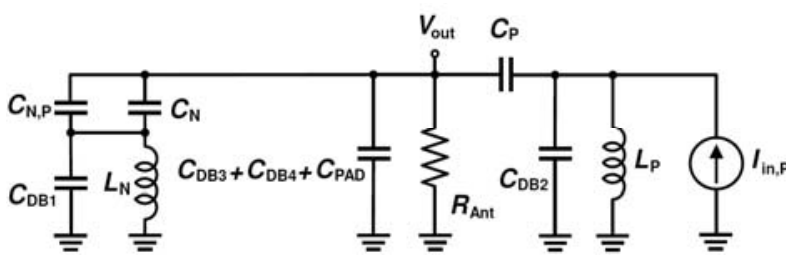

Figure 5. Half circuit model of the positive pulse generator

parallel with it, shown in Fig. 3. The combination of $\mathrm{C}_{\mathrm{N}, \mathrm{P}}+\mathrm{C}_{\mathrm{N}}$ and $\mathrm{L}_{\mathrm{N}}$ forms a series LC network oscillating at $\omega_{\mathrm{o}}=1 / \operatorname{sqrt}\left[\mathrm{L}_{\mathrm{N}} *\left(\mathrm{C}_{\mathrm{N}, \mathrm{P}}+\mathrm{C}_{\mathrm{N}}\right)\right]$. If $\mathrm{C}_{\mathrm{N}, \mathrm{P}}$ is chosen properly this network can diminish the power of signal around $1.6 \mathrm{GHz}$ frequency band. Contrarily, a complementary impulse can be applied to the negative half circuit. Similar to the positive half circuit, same phenomena exist for negative half circuit that creates an oscillation using $\mathrm{L}_{\mathrm{N}}$ and $\mathrm{C}_{\mathrm{P}}$, and the $\mathrm{C}_{\mathrm{P}, \mathrm{N}}$ and $\mathrm{C}_{\mathrm{P}}$ are used for filtering lower frequency components. Considering that $\mathrm{C}_{\mathrm{N}, \mathrm{P}}$ should not be present in the negative half circuit, a switch, $\mathrm{S}_{1}$, is used to disconnect it, while $S_{2}$ makes $C_{P}$ and $C_{P, N}$ in parallel.

Knowing the above-mentioned concepts, the pulse generator can be implemented using CMOS technology. Demonstrated in Fig. 4(a), impulse sources are realized using MOSFETs triggered by glitches. Also $M_{3}$ and $M_{4}$ are utilized instead of $S_{1}$ and $S_{2}$, respectively. Figure 4(b) shows the positive and negative glitch generator circuits. The delay modules can be tuned in order to control the width of the glitches and therefore to adjust the power of pulses. Moreover, by setting the "OOK_Enabler" input to '1', the modulation will be converted to OOK because $\mathrm{V}_{\text {GlitchN }}$ and $\mathrm{V}_{\text {CompN }}$ will be deactivated and the glitches are applied only to positive half circuit. 
TABLE I

Performance summary of the proposed pulse generator and comparison

\begin{tabular}{|c|c|c|c|c|c|c|}
\hline Specification & Praveen [15] & $\mathrm{Na}[7]$ & Muhr [16] & Xia [17] & Qin [18] & This Work \\
\hline Supply Voltage & $1.8 \mathrm{~V}$ & $1.2 \mathrm{~V}$ & $1.2 \mathrm{~V}$ & $1.2 \mathrm{~V}$ & $1.2 \mathrm{~V}$ & $1.2 \mathrm{~V}$ \\
\hline Energy consumption (pJ/Pulse) & 86 & 30 & 393 & 44 & 33 & 20 \\
\hline Data Rate & $250 \mathrm{MHz}$ & $200 \mathrm{MHz}$ & $10 \mathrm{MHz}$ & $100 \mathrm{MHz}$ & $1.3 \mathrm{Gbps}$ & $660 \mathrm{Mbps}$ \\
\hline -10dB Bandwidth (GHz) & $3.5-6.5$ & 0.5 & 1.8 & $3-5$ & $3.1-10.6$ & $3.3-6.2$ \\
\hline Output Amplitude & $0.5 \mathrm{~V}$ & $0.4 \mathrm{~V}$ & $1.72 \mathrm{~V}$ & $0.24 \mathrm{~V}$ & $86 \mathrm{mV}$ & $1 \mathrm{~V}$ \\
\hline Peak PSD Power (dBm/MHz) & -42 & - & - & -40 & - & -40.4 \\
\hline Process (nm) & 180 & 65 & 130 & 130 & 130 & 130 \\
\hline
\end{tabular}

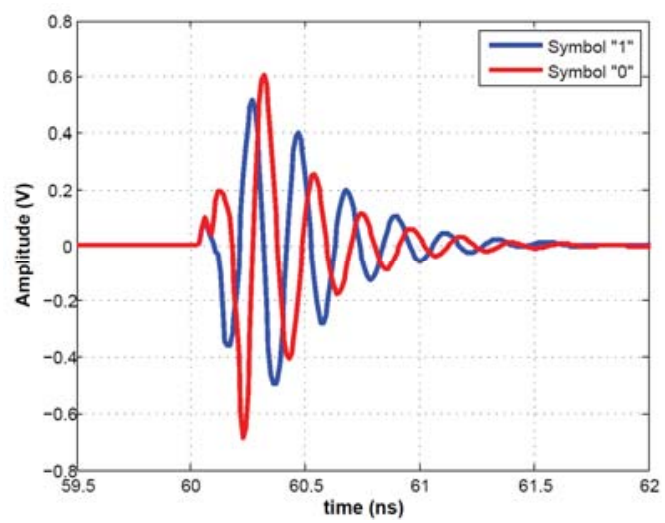

Figure 6. UWB pulse signals in time domain

Positive half circuit model of the pulse generator is shown in Fig. 5. Since the capacitance value of $C_{N}$ and $\mathrm{C}_{\mathrm{P}}$ are too small and comparable to drain-bulk parasitics added by $\mathrm{M}_{1-4}$, they degrade the frequency response of the circuit. Besides, adding a pad of the size 100 um $\times 100$ um to the output node exacerbates it. Therefore, in order to compensate such undesired effects a series $\mathrm{LC}$ filter, including $\mathrm{L}_{\mathrm{f}}$ and $\mathrm{C}_{\mathrm{f}}$, with resonance frequency around GPS band has been utilized.

\section{Simulation Results}

The proposed transmitter is implemented by 0.13 um CMOS process with $1.2 \mathrm{~V}$ supply voltage. BPSK modulation needs output pulses with $180^{\circ}$ phase shift, shown in Fig. 6. If all input data is '1', the pulse amplitude is $1 \mathrm{~V}$ and the overall power consumption of the pulse generator is only $1.9 \mathrm{~mW}$, including $160 \mathrm{uW}$ of the glitch generator circuit at $100 \mathrm{MHz}$ pulse repetition frequency (PRF). On the other hand, if all input data is ' 0 ', at the same clock rate the pulse amplitude is $1.28 \mathrm{~V}$ and the power dissipation is $2 \mathrm{~mW}$, resulting in $20 \mathrm{pJ} /$ pulse energy consumption. The large amplitude of the output signals provides mask satisfaction by means of a single UWB pulse. Thus, the $20 \mathrm{pJ} /$ pulse energy can be interpreted as the amount of power consumption per bit (20 pJ/pulse/bit). Illustrated

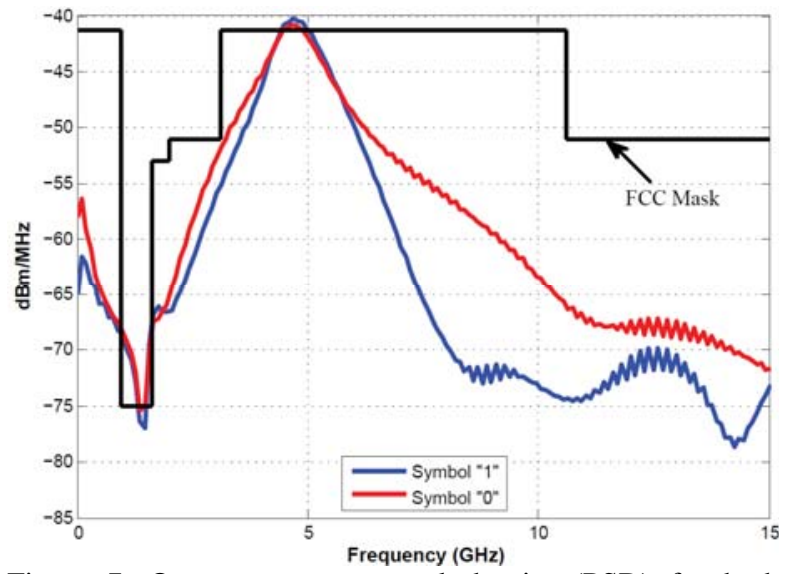

Figure 7. Output power spectral density (PSD) for both positive and negative signals

in Fig. 7, both impulses have central frequency of 4.8 GHz. Using periodogram, PSD of the output signal has been calculated for 10 cycles of it where the window size has been chosen such that it roughly contains one period of UWB pulses (10.24 ns). The power of pulses can be controlled by the delay module in glitch generator circuit. The wider the glitches are, the larger the value of current spikes charging $\mathrm{L}_{\mathrm{N}}$ or $\mathrm{L}_{\mathrm{P}}$ inductors. Figures 9(a) and 9(b) shows the glitches applied to $M_{1}$ and $\mathrm{M}_{2}$, respectively. For a glitch as wide as $45 \mathrm{ps}$ the peak power of pulses are around $-40.4 \mathrm{dBm} / \mathrm{MHz}$. The UWB pulse which corresponds to symbol '1' has $-10 \mathrm{~dB}$ bandwidth of 3.5-6 GHz, while the one for symbol '0' shows slightly larger bandwidth 3.3-6.2 GHz. Duration of each pulse is $1.5 \mathrm{~ns}$ which means the maximum PRF could be around $660 \mathrm{MHz}$. The performance summary of the proposed pulse generator along with a comparison with other published papers is listed in TABLE

\section{Conclusion}

A pulse generator with very high output amplitude (roughly as large as supply voltage) for BPSK modulation was presented. It achieves power consumption of $20 \mathrm{pJ}$ per pulse while PSD estimation 
shows each pulse can satisfy the FCC mask and can be considered as one bit. Due to the loss of bond-wires and compensating the effects of process corners, the glitch generator circuit has been designed such that the PSD peak power $(-40.4 \mathrm{dBm} / \mathrm{MHz})$ can be tuned and controlled. Thanks to the PSD similarity of both "0" and "1" symbols, designing the low-noise amplifier at the receiver will not be complex and it needs to be designed for $3 \mathrm{GHz}$ bandwidth with central frequency of $4.8 \mathrm{GHz}$. The proposed pulse generator also allows the communication using OOK modulation if required.

\section{Acknowledgment}

Project "NanoSTIMA: Macro-to-Nano Human Sensing: Towards Integrated Multimodal Health Monitoring and Analytics/NORTE-01-0145-FEDER000016" is financed by the North Portugal Regional Operational Programme (NORTE 2020), under the PORTUGAL 2020 Partnership Agreement, and through the European Regional Development Fund (ERDF).

\section{References}

[1] F. C. Commission et al., "In the matter of revision of part 15 of the commission's rules regarding ultra-wideband transmission systems," First Report and Order in ET Docket 98-153, 2002.

[2] A. Safarian and P. Heydari, Silicon-based RF Front-ends for ultra Wideband Radios. Springer Science \& Business Media, 2007.

[3] H. Nikookar and R. Prasad, Introduction to ultra wideband for wireless communications. Springer Science \& Business Media, 2008.

[4] M. Baghaei Nejad, "Ultra wideband impulse radio for wireless sensing and identification," 2008.

[5] A. Al Zaman and N. Islam, "Modulation schemes and pulse shaping in ultra-wideband," in Southeastcon, IEEE. IEEE, 2008, pp. 142-146.

[6] B. Razavi, Design of Analog CMOS Integrated Circuits, scond ed. McGraw-Hill, 2017.

[7] K. Na, H. Jang, H. Ma, Y. Choi, and F. Bien, “A 200$\mathrm{mb} / \mathrm{s}$ data rate 3.1-4.8-ghz ir-uwb all-digital pulse generator with db-bpsk modulation," IEEE Transactions on Circuits and Systems II: Express Briefs, vol. 62, no. 12, pp. 11841188, 2015.
[8] S. V. Mir-Moghtadaei, A. Fotowat-Ahmady, A. Z. Nezhad, and W. A. Serdijn, "A 90 nm-cmos ir-uwb bpsk transmitter with spectrum tenability to improve peaceful uwb-narrowband coexistence," IEEE Transactions on Circuits and Systems I: Regular Papers, vol. 61, no. 6, pp. 1836-1848, 2014.

[9] J. Zhao, C. Maxey, A. Narayanan, and S. Raman, "Cmos wideband pulse generators for uwb transmitter applications," in Sarnoff Symposium, IEEE, 2006, pp. 1-4.

[10] K. A. Al-Khateeb and A. R. Mohammed, "A novel design of 4-gbps and 6-gbps ir-uwb pulse generator," in Communication Software and Networks (ICCSN), IEEE 3rd International Conference on. IEEE, 2011, pp. 521-523.

[11] B. Schleicher, C. A. Ulusoy, and H. Schumacher, "A biphase modulator circuit for impulse radio-uwb applications," IEEE microwave and wireless components letters, vol. 20, no. 2, pp. 115-117, 2010.

[12] J. Radic, A. Djugova, L. Nagy, M. Videnovic-Misic, and L. Zivanov, "A novel low-complexity bpsk ir-uwb pulse generator in 0.13 um cmos technology," in Microelectronics Proceedings-MIEL, 29th International Conference on. IEEE, 2014, pp. 413-416.

[13] M. N. Karim, S. I. Hossain, and P. K. Saha, "A high data rate $130 \mathrm{~nm}$ cmos ir-uwb transmitter for inter-chip wireless communication," in Electrical \& Computer Engineering (ICECE), 7th International Conference on. IEEE, 2012, pp. 434-437.

[14] Z. Lin, W. Lei, and K. Li, "Design of adtr-dsss-ir-uwb transmitter for bpsk modulation," in Communications and Intelligence Information Security (ICCIIS), International Conference on. IEEE, 2010, pp. 236-239.

[15] P. Gunturi, N. W. Emanetoglu, and D. E. Kotecki, "A 250-mb/s data rate ir-uwb transmitter using current-reused technique," IEEE Transactions on Microwave Theory and Techniques, 2017.

[16] E. Muhr, R. Vauche, S. Bourdel, J. Gaubert, O. R. Sparrow, N. Dehaese, I. Benamor, and H. Barthelemy, "High output dynamic uwb pulse generator for bpsk modulations," in Ultra-Wideband (ICUWB), 2013 IEEE International Conference on. IEEE, 2013, pp. 170-174.

[17] L. Xia, Y. Huang, and Z. Hong, "Low power amplitude and spectrum tunable ir-uwb transmitter," Electronics Letters, vol. 44, no. 20, pp. 1200-1201, 2008.

[18] B. Qin, H. Chen, X. Wang, A. Wang, Y. Hao, L. Yang, and B. Zhao, "A single-chip 33pj/pulse 5th-derivative gaussian based ir-uwb transmitter in $0.13 \mu \mathrm{m}$ cmos," in Circuits and Systems, 2009. ISCAS 2009. IEEE International Symposium on. IEEE, 2009, pp. 401-404. 